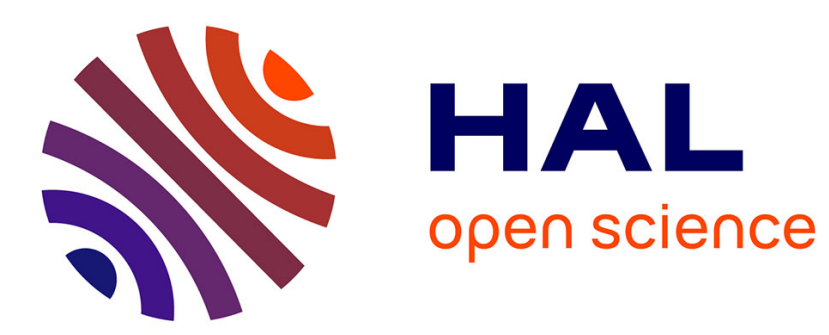

\title{
Predicting the Post-Impact Velocity of a Robotic Arm via Rigid Multibody Models: an Experimental Study
}

Ilias Aouaj, Vincent Padois, Alessandro Saccon

\section{To cite this version:}

Ilias Aouaj, Vincent Padois, Alessandro Saccon. Predicting the Post-Impact Velocity of a Robotic Arm via Rigid Multibody Models: an Experimental Study. 2020. hal-02434909v2

\section{HAL Id: hal-02434909 \\ https://hal.science/hal-02434909v2}

Preprint submitted on 15 Oct 2020 (v2), last revised 31 Mar 2021 (v4)

HAL is a multi-disciplinary open access archive for the deposit and dissemination of scientific research documents, whether they are published or not. The documents may come from teaching and research institutions in France or abroad, or from public or private research centers.
L'archive ouverte pluridisciplinaire HAL, est destinée au dépôt et à la diffusion de documents scientifiques de niveau recherche, publiés ou non, émanant des établissements d'enseignement et de recherche français ou étrangers, des laboratoires publics ou privés. 


\title{
Predicting the Post-Impact Velocity of a Robotic Arm via Rigid Multibody Models: an Experimental Study
}

\author{
Ilias Aouaj ${ }^{1}$, Vincent Padois ${ }^{2}$, Alessandro Saccon ${ }^{3 *}$
}

\begin{abstract}
Accurate post-impact velocity predictions are essential in developing impact-aware manipulation strategies for robots, where contacts are intentionally established at nonzero speed mimicking human manipulation abilities in dynamic grasping and pushing of objects. Starting from the recorded dynamic response of a 7DOF torque-controlled robot that intentionally impacts a rigid surface, we investigate the possibility and accuracy of predicting the post-impact robot velocity from the pre-impact velocity and impact configuration. The velocity prediction is obtained by means of an impact map, derived using the framework of nonsmooth mechanics, that makes use of the known rigid-body robot model and the assumption of a frictionless inelastic impact.

The main contribution is proposing a methodology that allows for a meaningful quantitative comparison between the recorded post-impact data, that exhibits a damped oscillatory response after the impact, and the post-impact velocity prediction derived via the readily available rigid-body robot model, that presents no oscillations and that is the one typically obtained via mainstream robot simulator software. The results of this new approach are promising in terms of prediction accuracy and thus relevant for the growing field of impactaware robot control. The recorded impact data (18 experiments) is made publicly available, together with the numerical routines employed to generate the quantitative comparison, to further stimulate interest/research in this field.
\end{abstract}

\section{INTRODUCTION}

State-of-the-art robot manipulation is performed by intentionally establishing contact at almost-zero velocity [1]. While this strategy is effective and provides guarantees regarding the successful accomplishment of the task, it also increases execution time as well as energy expenditure due to the associated acceleration/deceleration phases required to bring the contact velocity to zero when compared with a scenario where contact is established at non-zero velocity. Modern robots, in particular torque-controlled robots, are more and more designed for physical interaction, by means of employing back drivable and compliant joints which provide an impact torque ltering functionality and a physical protection to the reduction drives [2], [3], [4].

This allows for the experimentation of dynamic contact tasks where contact is established at non-zero velocity, leading

This work was partially supported by the Research Project I.AM. through the European Union H2020 program under GA 871899

${ }^{1}$ Ilias Aouaj has conducted this work during his MSc project at the Department of Mechanical Engineering, Eindhoven University of Technology (TU/e), The Netherlands (aouajilias@gmail.com)

${ }^{2}$ Vincent Padois is with Auctus, Inria - IMS (Univ. Bordeaux / Bordeaux INP / CNRS UMR 5218), 33405 Talence, France (vincent.padoiseinria.fr)

${ }^{3 *}$ Alessandro Saccon (corresponding author) is with the Department of Mechanical Engineering, Eindhoven University of Technology (TU/e), The Netherlands (a.saccon@tue.nl) to collisions which cause rapid changes in the system velocities and short-lived post-impact vibrations.

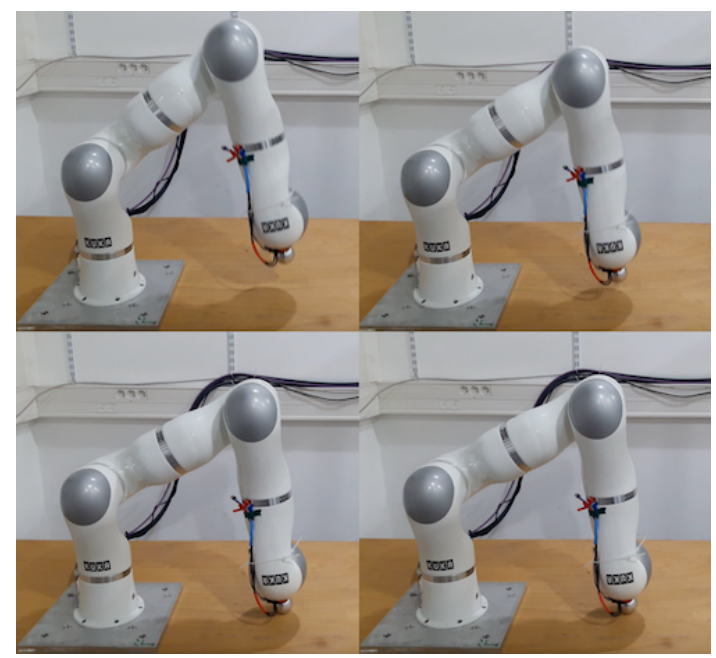

Fig. 1. Example of a vertical impact test at $0.2 \mathrm{~m} / \mathrm{s}$ conducted on a KUKA LWR IV+ robotic arm. The figure shows four snapshots from a recorded video. Time evolves from left to right and from top to bottom.

To achieve complex dynamic contact tasks, in parallel to adequate compliant mechanical design, it is necessary to develop a coherent framework that encompasses control, learning, planning, and sensing strategies in the presence of impacts and sudden velocity jumps [5], [6]. These methods assume to have at hand reliable forecast of the effects of impact phenomena to be able to cope with expected (and unexpected) impacts and ensure stability and desired level of performance while executing impact tasks.

Given the availability of new generation robots and everlasting desire/need to increase motion capabilities of robotic systems, the development of impact models is steadily gaining importance in the robotics community [7], together with the need for validation of these models against real experiments to assess their prediction ability and range of applicability [8]. One can speculate that collision exploitation will become as important in robotics as traditional collision avoidance.

Robot-environment collision models have a long history in robotics [9], [10], [11], [12], [13]. Despite this long history, experimental validation of these collision models is currently limited to robot locomotion [14], single free-falling objects (typically, lightweight small spherical objects, cylinders, or dumbbells) impacting a rigid surface or a robot manipulator, but with limited/no effect on the robot dynamics [15]. We are also unaware of publicly available robot-object-environment 
impact motion databases.

Acknowledging the emergence of robots capable of dynamic physical interactions, provided with high-frequency joint torque sensors and high-resolution encoders, the long term vision of our investigation is instead that of impactaware manipulation: we envision robots grabbing and pushing massive objects that have a fast and direct influence on the robot dynamics right after contact is established. In this context, it is fundamental to be able to predict, both from a planning and control point of view, post-impact velocities [16]. This, together with the creation of a publicly available repository of robot-object-environment impact motions, is also the goal of a recently started H2020 EU projec ${ }^{1}$.

The aim of this paper is to fill in some of the gaps of the robotic literature identified above, first by making available ${ }^{2}$ the joint data of the torque-controlled robotic arm while it impacts a wooden table at different speeds and impact angles (see Figure 1 for an illustration of this impact scenario) and then proposing a framework for quantitatively assessing the prediction ability of an impact map, derived from a known rigid body robot model, in determining the post-impact robot velocity.

As known from the field of nonsmooth mechanics, the impact map is the result of the combination of an impact equation and an impact law. For a robot manipulator, the impact equation is derived from an identified rigid body model or readily derived from $3 \mathrm{D}$ CAD design. When performing impact experiments, however, clear oscillatory transients are visible: in our experiments, these last for about a hundred milliseconds. At first, it is unclear how to assess the postimpact prediction performance of an impact map derived from an idealized algebraic impact law with an idealized rigid-body robot model (the one available with state-of-the-art robot identification techniques [17], [18]) versus the measured impact response. Hence, the main contribution of this work is to propose a methodology to separate the rigid response from the oscillatory response of the robot and consequently a methodology to assess the quality of the post-impact prediction. Such a post-impact velocity prediction has value for planning robot-object motions with impacts as well as for developing collision monitoring strategies to discriminate between planned and unplanned collisions. The procedure is illustrated first on an academic example in one dimension and then applied to the recorded impact data of a 7DOF robotic arm, showing good prediction capability.

The paper is organized as follows. Besides this introduction, Section II provides basic background information regarding nonsmooth mechanics and impact maps. A description of the impact experiments and problem statement is provided in Section III. Section IV details the main contribution, which is first introduced for an easily accessible academic example.

\footnotetext{
${ }^{1}$ Impact-Aware Manipulation by Dexterous Robot Control and Learning in Dynamic Semi-Structured Logistic Environments. Project Website: https://i-am-project.eu

${ }^{2}$ The impact data and associated MATLAB scripts (that make use of the Robotics Toolbox [19] for handling of the robot kinematics), can be downloaded from the following URL: www.dct.tue.nl/asaccon/ impact_dataset.zip
}


Fig. 2. Joint velocity signals for an impact at $0.2 \mathrm{~m} / \mathrm{s}$ and $90 \mathrm{deg}$ with respect to the horizontal table (indices from 1 to 3 are for the shoulder, 4 for the elbow, and 5 to 7 for the wrist). The vertical red line corresponds to the estimated impact time. Joint velocities are obtained via finite difference of the recorder encoder data.

Application of the proposed methodology on the 7DOF robot data is presented in Section $V$. Finally, conclusions and future work is discussed in Section VI.

\section{NONSMOOTH MECHANICS IMPACT MAPS}

Within dynamical systems theory, nonsmooth mechanics [20] is quite a mature theoretical framework that combines rigid body modeling with algebraic impact laws, with the aim of capturing the post-impact state of a mechanical system based on the ante-impact configuration and velocity. The essential modeling assumption within this framework is a space-and-time scale separation between the contact and body dynamics that justifies approximating the impact dynamics as instantaneous (i.e., taking zero time) and consequently allowing for instantaneous jumps in the system's velocity and corresponding impulsive contact forces.

While admitting instantaneous velocity jumps and impulsive contact forces is a clear idealization of the contact dynamics (for the family of robots we are considering in this work, impact duration is typically in the range of 5 to 10 $\mathrm{ms}$ as shown in [21], [22], [23]), advanced impact models can provide impressive prediction capabilities even in the presence of multiple simultaneous impacts [24]. Also, these algebraic impact models have demonstrated extremely effective in planning and control for mechanical systems undergoing impacts, going from the estimation of the distribution of 
possible poses of a known object dropped on a surface from an arbitrary height [25], model-based dynamic robot locomotion [26], [27], and accurate batting of flying objects [15]. There is therefore good hope that similar models, once validated, can be also of great use in impact-aware robot manipulation.

In the simplest form assumed in this paper, the impact map (i.e., the post-impact velocity prediction) is obtained starting from the standard equations of motion

$$
\begin{array}{ll}
\mathbf{M}(\mathbf{q}) \ddot{\mathbf{q}}+\mathbf{h}(\mathbf{q}, \dot{\mathbf{q}})=\boldsymbol{\tau}+\mathbf{J}_{\mathbf{N}}^{T}(\mathbf{q}) \lambda_{N}, & \text { if } g_{N}(\mathbf{q})=0, \\
\mathbf{M}(\mathbf{q}) \ddot{\mathbf{q}}+\mathbf{h}(\mathbf{q}, \dot{\mathbf{q}})=\boldsymbol{\tau}, & \text { if } g_{N}(\mathbf{q})>0
\end{array}
$$

where $\mathbf{q} \in \mathbb{R}^{n}$ are the generalized coordinates, $M$ the mass matrix, $\mathbf{h}$ the Coriolis, centrifugal, and gravity terms, $\tau$ the actuation torque, $g_{N}$ the gap function representing the distance between the robot and the contact surface (obtainable via forward kinematics), $\lambda_{N} \in \mathbb{R}$ is the normal contact force, and $\mathbf{J}_{N}(\mathbf{q}) \in \mathbb{R}^{1 \times n}$ is the corresponding contact Jacobian $\left(\mathbf{J}_{N}(\mathbf{q}):=\partial g_{N} / \partial \mathbf{q}\right)$. In nonsmooth mechanics, $\lambda_{N}$ is allowed to become impulsive at the moment of collision and this leads to the so-called impact equation [20]

$$
\mathbf{M}(\mathbf{q})\left(\dot{\mathbf{q}}^{+}-\dot{\mathbf{q}}^{-}\right)=\mathbf{J}_{N}^{T}(\mathbf{q}) \Lambda_{N}
$$

where $\Lambda_{N}$ represents the impulsive force magnitude and $\dot{\mathbf{q}}^{+}$ and $\dot{\mathbf{q}}^{-}$denote the post- and ante-impact joint velocities, respectively. The impact map is obtained combining the impact equation with an impact law that in case of a frictionless inelastic impact as we consider in this work reads

$$
\dot{g}_{N}^{+}=\mathbf{J}_{N}(\mathbf{q}) \dot{\mathbf{q}}^{+}=0 .
$$

The equation above is simply stating that the normal component of the Cartesian post-impact velocity of the end effector should be zero after the impact. The impact map allows for a velocity jump, while keeping the configuration unaltered. The combination of (3) and (4) allows to express $\Lambda_{N}$ as a function $\dot{\mathbf{q}}^{-}$and this, by substituting this expression in (3), to predict the value of $\dot{\mathbf{q}}^{+}$. One obtains therefore the following singlepoint frictionless inelastic impact map, valid for $g_{N}(\mathbf{q})=0$,

$$
\dot{\mathbf{q}}^{+}=\left(\mathbf{I}-\mathbf{M}^{-1} \mathbf{J}_{N}^{T}\left(\mathbf{J}_{N} \mathbf{M}^{-1} \mathbf{J}_{N}^{T}\right)^{-1} \mathbf{J}_{N}\right) \dot{\mathbf{q}}^{-} .
$$

In the presence of friction, partially elastic and/or multiple simultaneous impacts, the formulation of the impact map becomes necessarily more sophisticated and often implicit [28] but this is not essential for transmitting the core message of this work and is therefore left out in this brief overview.

\section{PROBLEM STATEMENT}

Several impact experiments have been conducted between a torque controlled robotic arm (KUKA LWR IV+) and a smooth wooden table (cf. Figure 1). The robot impacted the table via a spherical metal probe that was secured with bolts to the standard robot tool mounting plate. A representative example of the recorded joint angles during an impact experiment is given in Figure 2. In this particular experiment, the impact between the table and the robot is normal to the table and occurs at a Cartesian velocity of $0.2 \mathrm{~m} / \mathrm{s}$, approximately at time $1.94 \mathrm{~s}$. A post-impact damped vibratory response can be observed, lasting approximately $100 \mathrm{~ms}$. Just the second, fourth, and sixth joint are notably affected by the impact: this is justified by the fact that the impact motion occurs essentially on a vertical plane (2D motion), which is also an approximate plane of symmetry for the robot and in which the mentioned joints are the ones that affect the arm motion the most (essentially, we are looking at a planar impact of a planar RRR manipulator).

Besides this particular experiment, various combinations of low impact velocities and angles have been recorded (about twenty experiments, with repetitions). Overall, the impact velocity varied between 0.1 and $0.2 \mathrm{~m} / \mathrm{s}$ and the impact angle between 30 and 90 degrees with respect to the table surface.

The impact experiments were obtained employing a taskbased QP robot controller ${ }^{3}$ (cf. [29] and [30] and references therein). The control torques are computed based on 7DOF rigid-body kinematics, velocity kinematics and dynamic models obtained using the KDL library ${ }^{4}$, The controller is assigned a pose task for the end-effector (with a linear motion for the metal probe center with constant velocity and constant orientation) and a regularization task (constant joint posture) to avoid self motions. The goal pose is located below the wooden table and cannot be reached as impact with the table occurs first. Once contact has been detected using torque measurements at the joint level, the controller switches to pure gravity compensation.

To validate the lack of influence of the controller in the impact response, different experiments have been performed where the Cartesian task PD gains have been altered verifying that no appreciable difference in the post-impact response in the joint signals could be observed. This leads to hypothesize that the post-impact oscillations (cf. Figure 2) are solely of structural nature or due to the high bandwidth low-level torque controllers active on the joints. A confirmation of this fact would require the mounting of accelerometers and accessing both motor and joint encoders on the robot: this is deemed as a future research but the observed oscillations are very likely due to joint level non-rigidities induced by the gears (Harmonic drive) of the transmission as well as the joint torque control technology. What is relevant for the discussion that follows is, in any case, that the system exhibits damped oscillatory modes whose time scales are of at least an order of magnitude higher than the impact phenomenon.

As anticipated in the introduction, the impact data reported in Figure 2 makes it apparent that there is a fundamental challenge when trying to employ a post-impact velocity prediction based on a rigid-body robot model. Post-impact predictions based on rigid-body models do not exhibit any oscillatory behavior after an impact and therefore it is unclear how their prediction can be validated against real impact experiments that present damped post-impact oscillatory transients. The challenge is therefore summarized in the following problem

3 https://github.com/kuka-isir/rtt_lwr/releases and https: //orca-controller.readthedocs.io/

4 https://github.com/orocos/orocos_kinematics dynamics 

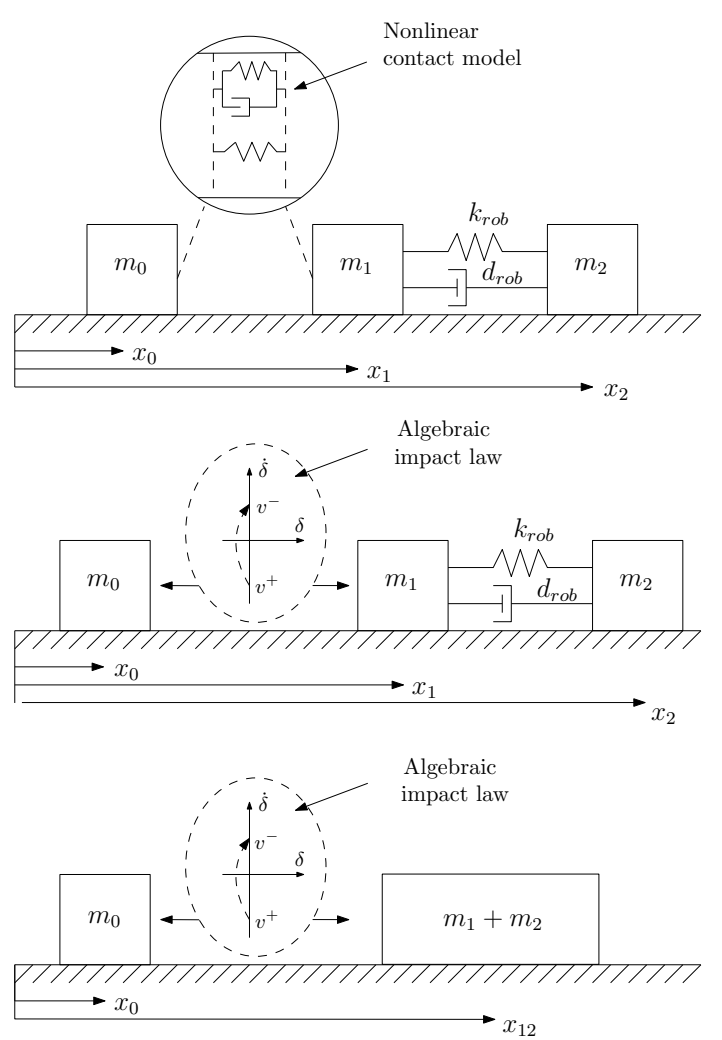

Fig. 3. Three levels of abstraction of an impact scenario, where a "1-DOF robot" (right) physically interacts with an external rigid object (left): (top) Contact is compliant and the robot is flexible; (middle) Contact is modelled via an algebraic impact law and the robot is flexible; (bottom) Contact is modelled via an algebraic impact law and the robot is rigid.

statement, for which we propose a solution in Section IV

Problem statement. How can experimental post-impact velocity data be quantitatively compared with post-impact velocity predictions, readily obtainable via an available rigidbody robot model and an algebraic impact law?

Note that post-impact velocity predictions based on rigidbody models are essentially what dynamic simulators (e.g., Bullet, ODE, or Vortex Studio) embedded in mainstream robot simulation suites such as Gazebo or CoppeliaSim (former VREP) provide, at least in simple impact scenarios.

\section{CONTRIBUTION}

In this section, we propose a procedure to assess the performance of the post-impact prediction obtainable with a rigid body impact model against the recorded impact data. At first, for illustration purposes, this procedure is introduced by means of an academic example employing two bodies colliding with each other along a straight line. We model these two bodies and the interaction between them in different ways, obtaining in total three different dynamical systems, as depicted in Figure 3. The purpose of this exercise is to show that a fully rigid model with nonsmooth impact law is capable of capturing the "steady state" response of more accurate and realistic flexible models and that by removing the flexibilityinduced transient response from the flexible models, the rigid model and the flexible ones can be quantitatively compared at impact time.

All these three models are made of an "interaction environment" (the body depicted on the left, considered fully rigid and with mass $m_{0}$ ) and a "robot" (the body depicted on the right, with total mass $m_{1}+m_{2}$ ). The interpenetration and relative velocity between the interaction environment and the body are denoted, respectively, as $\delta$ and $v$. Note that $\delta=-g_{N}$, with $g_{N}$ the gap function as in Section [I] The three models are detailed in the following bullets.

- Model A: Compliant-contact/flexible-robot. In this model, the robot (right) is assumed to be flexible and represented as the ensemble of two masses $\left(m_{1}\right.$ and $\left.m_{2}\right)$ connected via a compliant coupling, with linear stiffness $k_{r o b}$ and damping $d_{r o b}$. The interaction between the robot and the environment (the mass $m_{0}$ on the left) is modeled via the Hunt-Crossley nonlinear contact model [31], which relates the interpenetration $\delta$ and its rate of change to the contact force $F$. Namely, we have

$$
F(\delta, \dot{\delta})= \begin{cases}k_{e n v} \delta^{c}+d_{e n v} \delta^{c} \dot{\delta}, & \delta \geq 0, \\ 0, & \delta<0,\end{cases}
$$

with $k_{e n v}, d_{e n v}$, and $c$ chosen constants. Due to the interpenetration-dependent damping coefficient $d_{e n v} \delta^{c}$, the Hunt-Crossly contact model does not exhibit any (nonphysical) contact force jump when contact is established at nonzero velocity. In the simulations presented hereafter in this section, the damping coefficient $d_{e n v}$ is set to a very high value in order to simulate (almost) inelastic impacts;

- Model B: Hard-contact/flexible-robot. In this model, the contact interaction between the (flexible) robot is modelled via the fully inelastic impact law $\dot{\delta}^{+}=\dot{x}_{1}^{+}-\dot{x}_{0}^{+}=0$, triggered whenever $\delta:=x_{1}-x_{0}=0$ and $\dot{\delta}^{-}=\dot{x}_{1}^{-}-\dot{x}_{0}^{-}<0$ (" + " and "-" denote left and right limits at impact time);

- Model C: hard-contact/rigid-robot. In this model, the robot is considered as a point mass (with mass $m_{1}+m_{2}$ ) and the robot-environment contact is rigid (same impact law as in Model B).

Looking at these three models, it should be apparent that model A is the closest one to physical reality, while model $\mathrm{C}$, based on rigid-body assumption, represents the one that is typically available and used for robot control and planning.

Illustrative numerical simulation of an impact. We consider the situation where the environment $\left(m_{0}\right)$ and the robot $\left(m_{1}\right.$ and $\left.m_{2}\right)$ start at rest and a constant force is applied to $m_{2}$. This force accelerates the robot towards the left until it impacts with the environment and a sudden velocity change is experienced, making the environment start moving and the robot to suddenly decelerate. In Figure 4, we report the corresponding motion of the robot for the three models described

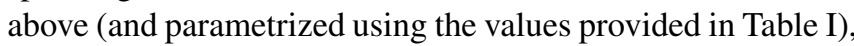
by showing the position and velocity signals of the two masses $m_{1}$ and $m_{2}$ (for model $\mathrm{C}$, the motion of $m_{1}$ and $m_{2}$ are identical as they are rigidly connected).

Without loss of generality, the initial separation between $m_{0}$ and $m_{1}$ was set to $0.4 \mathrm{~m}$ and the constant pushing force 

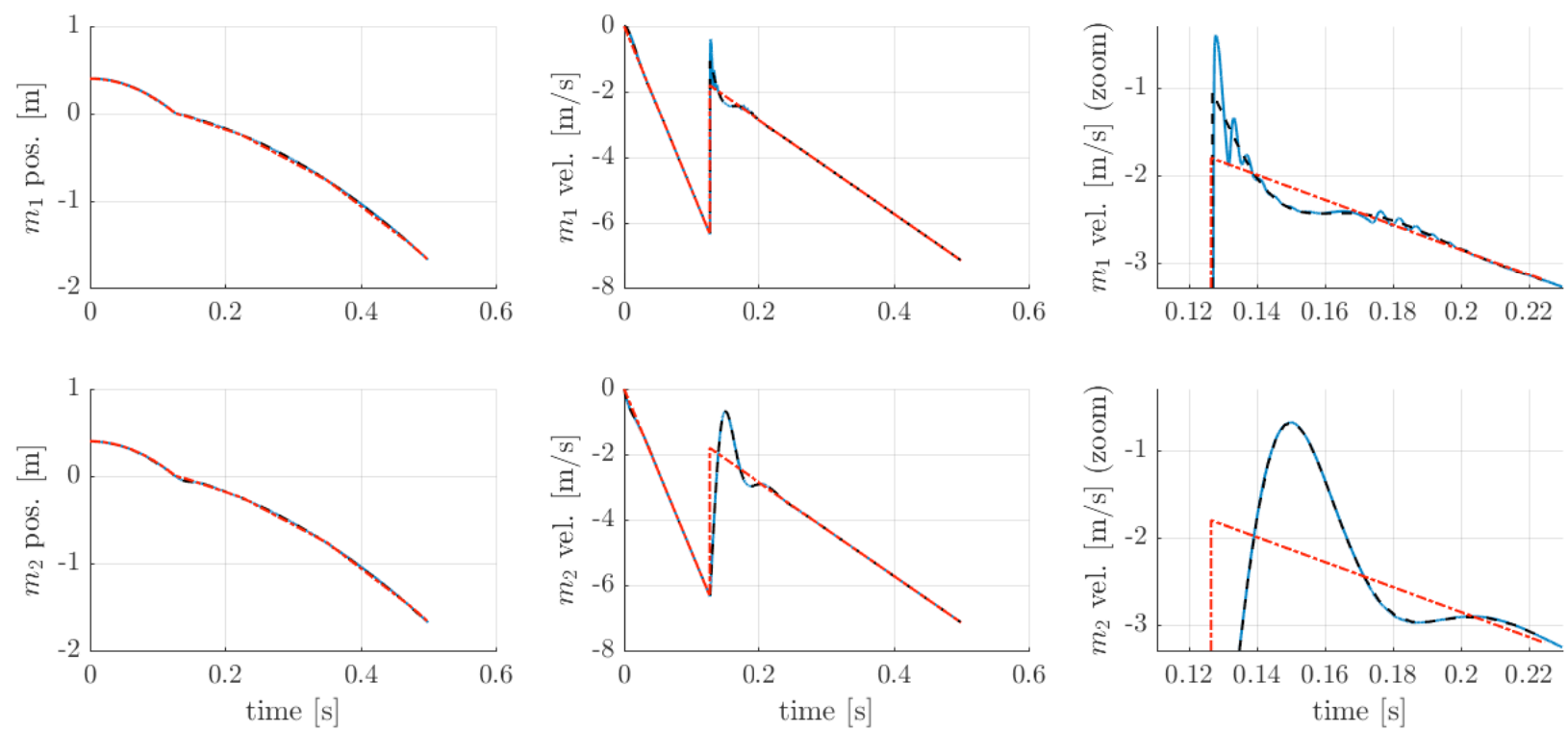

Fig. 4. Simulation results corresponding to the three models, where motion signals are given for mass $m_{1}$ and $m_{2}$. The first column shows the position of mass $m_{1}$ (top) and $m_{2}$ (bottom). The second column shows the velocity of mass $m_{1}$ (top) and $m_{2}$ (bottom). The third column is a zoomed version of the second column, about the impact time. Model A (compliant-contact/flexible-robot) is depicted in blue, Model B (hard-contact/flexible-robot) in dashed black, and Model C (hard-contact/rigid-robot) in dash-dot red.

TABLE I

PARAMETERS USED IN THE NUMERICAL SIMULATIONS

\begin{tabular}{l|l|l||l|l|l} 
Parameter & Value & Units & Parameter & Value & Units \\
\hline$m_{0}$ & 5 & $\mathrm{~kg}$ & $m_{1}$ & 1 & $\mathrm{~kg}$ \\
$m_{2}$ & 1 & $\mathrm{~kg}$ & $c$ & $3 / 2$ & {$[-]$} \\
$k_{\text {env }}$ & $1 \cdot 10^{8}$ & $\mathrm{~N} / m^{c}$ & $d_{\text {env }}$ & $1 \cdot 10^{8}$ & $\mathrm{Ns} / \mathrm{m}^{c+1}$ \\
$k_{\text {rob }}$ & $1 \cdot 10^{4}$ & $\mathrm{~N} / \mathrm{m}$ & $d_{\text {rob }}$ & 80 & $\mathrm{~N} \mathrm{~s} / \mathrm{m}$
\end{tabular}

to $100 \mathrm{~N}$. The simulation parameters for the Hunt-Crossley model $\left(k_{e n v}, d_{e n v}\right.$, and $\left.c\right)$ have been chosen based on the metal-wood interaction in the real robot-table experiment, assuming a stiffness corresponding to hard wood, a spherehalfspace Hertz contact, and high damping to represent an impact with a small coefficient of restitution. The robot stiffness $k_{r o b}$ is chosen to be lower than the contact's and damping $d_{r o b}$ is selected to get a lightly damped response. Simulations were performed in MATLAB using standard ODE solvers (a variable-order stiff solver, ode $15 \mathrm{~s}$, is used to deal with the stiff ODE related to high contact stiffness used in model A).

Discussion about the simulation results - The numerical results on Figure 4 show that the dynamic responses of robot in models A and B are essentially indistinguishable at the time scale of interest. This is simply an illustration of the timeand-space scale separation of contact and body dynamics, which justify the contact modeling simplification employed in nonsmooth mechanics. The zoomed-in version of the impact response of $m_{1}$ depicted in the last column of Figure 4 shows that the compliant and nonsmooth rigid contact models do indeed differ on a millisecond time scale, with the nonsmooth model $\mathrm{B}$ just capturing the average response of the compliant model A. The numerical simulation of model A shows explicitly two distinctive dynamics at work: one fast due to contact stiffness $(0.12-0.14 \mathrm{~s}$ in the top subplot) and the one slower, captured also by model B $(0.12-0.21 \mathrm{~s}$ and beyond). Inspection of the gap function actually shows bouncing of mass $m_{1}$ on $m_{0}$ before full adhesion, a phenomenon that is comparable with foot chattering in legged locomotion [32]. Other simulations with higher damping at contact (namely, $1 \cdot 10^{9} \mathrm{Ns} / \mathrm{m}^{c+1}$ ), instead, show no bouncing between $m_{1}$ and $m_{0}$ and an even closer matching between the motions of mass $m_{1}$ for models A and B (these plots are not reported here for space limitation, but they are easily reproduced with the provided MATLAB script).

Interesting to note is that the responses of model A and model B at the "actuation-and-position-sensing" side (i.e., $m_{2}$ ) are essentially indistinguishable, as clearly shown in the bottom middle and right plots of Figure 4

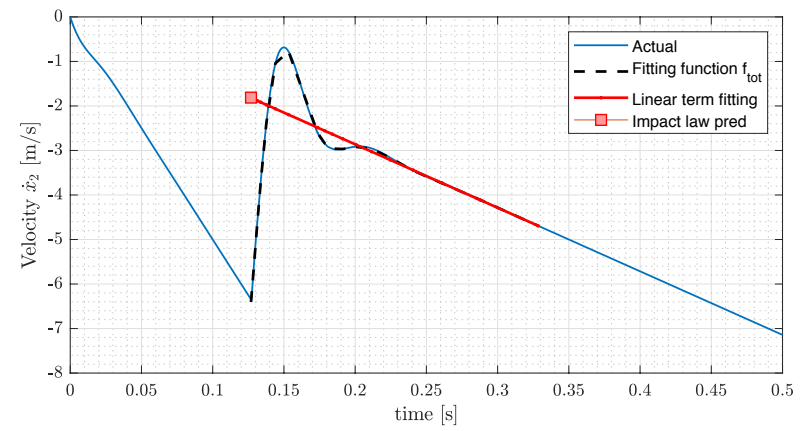

Fig. 5. Numerical data fitting result. Here we used the fitting scheme on the velocity signal of mass $m_{2}$, where the linear term of the fitting function is extrapolated back to the time instance of impact. In addition, the prediction of the post-impact velocity of the impact map is also provided, which is compared to the estimation provided by the extrapolation. 
Key observation and contribution. At this point, it becomes interesting also to compare the behavior of model B (flexible robot) with that of model C (rigid robot). In particular, regarding the actuation-and-sensing side of the robot $\left(m_{2}\right)$. Figure 4 illustrates that the velocity of the flexible robot model converges to that of the rigid robot model in about $100 \mathrm{~ms}$, suggesting to interpret the response of the flexible robot model as that of a low-pass filter acting on a velocity step input. The flexible robot dynamics after an impact are thus seen as a faster dynamics than that of the gross motion of the bodies after impact. Given the time-and-space scales of these vibrations (in real experiments, these vibrations are only observable looking at encoder data), we suggest to treat the post-impact vibration dynamics as linear and thus as the superposition of exponentially decaying oscillatory responses (including constant and linear terms corresponding to a step input). Least-squares fitting/modal analysis techniques can thus be used to remove the oscillatory part of the post-impact time response, extracting just the steady-state response (sum of a constant and a linear term as a function of time).

An example of application of this procedure, where the velocity signal is decomposed into the sum of a constant, a linear function of time, and just one exponentially decaying function of the form $A \exp (\omega t+\phi)$ is given in Figure 5 .

Proposed quantitative comparison procedure. Summarizing, we propose the following method to quantitatively compare experimental post-impact data with post-impact velocity predictions derived from a rigid multi-body impact map:

1) Identify the impact time $t_{i}$ in the experimental data by looking at, e.g., sharp variations of joint encoder data (either visually as we did in this work or by the use of automatic methods such as those described in [33] and references therein);

2) Extract the impact robot configuration $\mathbf{q}\left(t_{i}\right)$ and corresponding pre-impact joint velocity $\dot{\mathbf{q}}^{-}\left(t_{i}\right)$ and compute the rigid-robot post-impact joint velocity estimate $\dot{\mathbf{q}}^{+}\left(t_{i}\right)$ employing the impact map (5);

3) Use (nonlinear) least squares fitting or frequencydomain-based procedures on the signal $\dot{\mathbf{q}}(t)$ to separate the affine (=constant plus linear) response from the oscillatory damped response ("sum of eigenmodes") over the interval $\left[t_{i}, t_{i}+T_{s}\right]$ where vibrations are observed (with $T_{s}$ the settling time). Employ the affine part to construct the virtual rigid-robot post-impact velocity $\hat{\dot{\mathbf{q}}}^{+}\left(t_{i}\right)$;

4) Evaluate the (relative and absolute) error between $\dot{\mathbf{q}}^{+}\left(t_{i}\right)$ and $\hat{\mathbf{q}}^{+}\left(t_{i}\right)$ for impacts occurring at different postures and velocities, to quantify the general accuracy of the post-impact velocity estimation (considering the virtual rigid-robot post-impact velocity $\hat{\mathbf{q}}^{+}\left(t_{i}\right)$ the ground truth obtained from experiments).

The procedure above can be employed in Cartesian space, rather than in joint space, by performing points 3 and 4 using the Cartesian velocities $\overrightarrow{\mathbf{v}}^{+}\left(t_{i}\right)=\mathbf{J}\left(\mathbf{q}\left(t_{i}\right)\right) \dot{\mathbf{q}}^{+}\left(t_{i}\right)$ and $\widehat{\mathbf{v}}^{+}\left(t_{i}\right)$, obtained by least squares fitting on the sig- nal $\mathbf{J}(\mathbf{q}(t)) \dot{\mathbf{q}}(t)$, in place of the joint velocities $\dot{\mathbf{q}}^{+}\left(t_{i}\right)$ and $\hat{\mathbf{q}}^{+}\left(t_{i}\right)$, respectively. The result of applying this method on the 7DOF KUKA arm are reported in the following section.

\section{FITTING RESULTS ON THE 7DOF ARM}

Figure 6 illustrates the result of the fitting procedure described in the previous section now applied to the 7DOF arm experimental data collected for an impact at $0.2 \mathrm{~m} / \mathrm{s}$ and three different impact angles. Only vertical and longitudinal Cartesian velocities are reported, as lateral displacement is negligible in the performed impact experiments as explained in Section IIII Cartesian velocities are obtained from the recorded joint velocities and end-effector Jacobian. For least squares fitting, we employ for each Cartesian velocity component the fitting function

$$
f_{\text {tot }}(t ; \mathbf{p}):=v^{-}+a t+A\left(e^{\gamma t} \cos (\omega t+\phi)-\cos (\phi)\right)
$$

where $t$ denotes the time after the impact $t_{i}, v^{-} \in \mathbb{R}$ the preimpact velocity, and $\mathbf{p}:=(a, A, \gamma, \omega, \phi) \in \mathbb{R}^{5}$ denotes the vector of parameters (slope $a$, amplitude $A$, decay factor $\gamma$, frequency $\omega$, and phase shift $\phi$ ) for the least-squares fitting.

More precisely, the full set of parameters is only used for the vertical Cartesian direction (which has always a clear dominant second order response with large amplitude) while for the fitting of the motion in the longitudinal Cartesian direction the frequency and decay rate are set equal to the one identified for the vertical Cartesian direction. In this way, we obtain a single real eigenmode to describe the oscillation in accordance with the post-impact linear oscillation assumption. The fitting procedure is applied on a $150 \mathrm{~ms}$ time window, that was selected based on the stabilization of the least squares fitting parameters and roughly corresponds to three oscillation periods. In Figure 6, the reconstructed virtual rigidbody response $v^{-}-A \cos (\phi)+a t$ is shown as a red line (left $z$, right $x$ Cartesian component). Its value $\hat{v}^{+}=v^{-}-A \cos (\phi)$ at impact time (the tip of the red line) should be compared with the post-impact velocity estimate $\vec{v}^{+}$(the red square), derived via the rigid-body impact map. The impact map is derived from the rigid-body robot model employed by the QP robot controller, combined with the frictionless inelastic impact law between the end-effector tip and the (assumed rigid) wooden table, as discussed in Section II

Overall, the reconstructed and rigid-body impact map predictions, $\hat{v}_{i}^{+}$and $\vec{v}_{i}^{+}, i=\{x, z\}$, are in very good agreement. As summarized in Table II this holds not just for the $0.2 \mathrm{~m} / \mathrm{s}$ impacts, but also for the impact experiments at $0.15 \mathrm{~m} / \mathrm{s}$ and $0.1 \mathrm{~m} / \mathrm{s}$. The table shows both the normal and longitudinal velocity as well as the absolute prediction error $\eta_{i}:=\mid \vec{v}_{i}^{+}-$ $\hat{v}_{i}^{+} \mid, i=\{x, z\}$, in $m / s$.

On average, we get a $8 \mathrm{~mm} / \mathrm{s}$ absolute error and $7.3 \%$ relative error on predicting post-impact sliding velocity (the relative error is computed as $\left.2\left|\vec{v}_{i}^{+}-\hat{v}_{i}^{+}\right| /\left|\vec{v}_{i}^{+}+\hat{v}_{i}^{+}\right|\right), i=$ $\{x, z\}$.

Looking at the measured impact response, it is noticeable that the assumption of a second order system type response is only partially valid and higher frequency modes currently not 



Fig. 6. Cartesian velocity fitting results for impact at $0.2 \mathrm{~m} / \mathrm{s}$ under angles ranging from 30 to 90 deg w.r.t. to the horizontal table. The x-direction (right) is tangential to the table and the z-direction (left) is normal. Note how the fully rigid-model impact law prediction (red square) agrees with the prediction resulting from filtering out the post-impact transient (left end point of the red segment)

TABLE II

EVALUATION OF THE IMPACT MAP AND FITTING RESULTS FOR THE TWO EXPERIMENTAL DATA SETS (DATA IN $m / s$ )

\begin{tabular}{l|lllllllllllllllll} 
velocity, angle & $\tilde{v}_{z}^{-}$ & $\hat{v}_{z}^{+}$ & $\hat{v}_{z}^{+}$ & $\eta_{z}$ & $\tilde{v}_{x}^{-}$ & $\hat{v}_{x}^{+}$ & $\hat{v}_{x}^{+}$ & $\eta_{x}$ & $\tilde{v}_{z}^{-}$ & $\hat{v}_{z}^{+}$ & $\hat{v}_{z}^{+}$ & $\eta_{z}$ & $\tilde{v}_{x}^{-}$ & $\hat{v}_{x}^{+}$ & $\hat{v}_{x}^{+}$ & $\eta_{x}$ \\
\hline \hline $0.10 \mathrm{~m} / \mathrm{s}, 30^{\circ}$ & -0.097 & 0 & -0.006 & 0.006 & 0.031 & 0.094 & 0.096 & 0.002 & -0.085 & 0 & -0.007 & 0.007 & 0.061 & 0.120 & 0.112 & 0.008 \\
$0.10 \mathrm{~m} / \mathrm{s}, 60^{\circ}$ & -0.085 & 0 & -0.009 & 0.009 & 0.061 & 0.122 & 0.109 & 0.013 & -0.099 & 0 & -0.003 & 0.003 & 0.028 & 0.088 & 0.094 & 0.006 \\
$0.10 \mathrm{~m} / \mathrm{s}, 90^{\circ}$ & -0.101 & 0 & -0.005 & 0.005 & 0.004 & 0.055 & 0.050 & 0.006 & -0.103 & 0 & -0.003 & 0.003 & -0.007 & 0.039 & 0.050 & 0.011 \\
$0.15 \mathrm{~m} / \mathrm{s}, 30^{\circ}$ & -0.175 & 0 & -0.007 & 0.007 & 0.064 & 0.176 & 0.181 & 0.005 & -0.120 & 0 & -0.012 & 0.012 & 0.092 & 0.178 & 0.170 & 0.008 \\
$0.15 \mathrm{~m} / \mathrm{s}, 60^{\circ}$ & -0.114 & 0 & -0.010 & 0.010 & 0.096 & 0.174 & 0.163 & 0.011 & -0.137 & 0 & -0.006 & 0.006 & 0.054 & 0.140 & 0.149 & 0.009 \\
$0.15 \mathrm{~m} / \mathrm{s}, 90^{\circ}$ & -0.148 & 0 & -0.003 & 0.003 & -0.006 & 0.072 & 0.064 & 0.008 & -0.148 & 0 & -0.002 & 0.002 & 0.002 & 0.072 & 0.072 & 0.000 \\
$0.20 \mathrm{~m} / \mathrm{s}, 30^{\circ}$ & -0.175 & 0 & -0.007 & 0.007 & 0.064 & 0.176 & 0.181 & 0.005 & -0.144 & 0 & -0.011 & 0.011 & 0.120 & 0.222 & 0.209 & 0.013 \\
$0.20 \mathrm{~m} / \mathrm{s}, 60^{\circ}$ & -0.165 & 0 & -0.005 & 0.005 & 0.108 & 0.223 & 0.204 & 0.019 & -0.168 & 0 & -0.004 & 0.004 & 0.086 & 0.190 & 0.190 & 0.000 \\
$0.20 \mathrm{~m} / \mathrm{s}, 90^{\circ}$ & -0.185 & 0 & 0.000 & 0.000 & 0.000 & 0.098 & 0.085 & 0.013 & -0.178 & 0 & -0.002 & 0.002 & 0.003 & 0.093 & 0.099 & 0.006
\end{tabular}

modeled are present. This is more noticeable at the joint level (results are reproducible with the provided MATLAB scripts) and therefore we have chosen here to limit the model analysis based on a single mode of vibration at Cartesian level. The use of a more sophisticated modal analysis procedure at joint level is considered as the next step of our investigation.

\section{CONCLUSIONS}

In this work, we have performed several impact experiments between a 7DOF torque-controlled robotic arm and a wooden table, at different velocities and impact angles. The proposed post-impact velocity prediction procedure, based on the idea of removing post-impact oscillation components, shows to be in very good agreement with a fully-rigid robot impact model. This procedure, which is not tailored to a specific robot, can be used to assess if a fully rigid-body impact model can provide reliable post-impact velocity prediction that are of use in impact-aware robot planning, control, and perception.

For the specific impact experiments performed with the KUKA LWR IV+, we obtained a $7.3 \%$ relative error and $8 \mathrm{~mm} / \mathrm{s}$ absolute error difference between the measured and predicted sliding velocity, over a set of 18 experiments. This good accuracy is rather surprising giving the fact that we are comparing an ideal rigid-body impact model with real experiments that contain unmodeled post-impact (structural) vibrations, that are "filtered out" by the proposed procedure. Further investigation is required to assess what level of accu- 
racy is required to achieve satisfying performance in impactaware manipulation for specific applications.

For the considered impact scenarios, the post-impact response in the vertical Cartesian direction clearly shows a dominant second-order-system type response to an impact. On the horizontal direction, the response is more complex and more advanced modal analysis techniques could be explored to assess if this could have an effect in the further reduction of the prediction error. We expect that least-square fitting procedures with multiple oscillatory modes can be borrowed from the structural dynamics literature to this end.

Further research will consider explicit inclusion of the joint flexibility and motor dynamics in the impact laws, several impact configurations and their influence in post-impact oscillations, different type of robot manipulators (possibly with additional or different compliant components, other than harmonic drives), oblique impacts with rough surfaces to study the postimpact velocity predictability in the present of surface friction, and complex impacts such as, e.g., simultaneous grabbing and lifting of heavy boxes with dual-arm systems, with the goal of employing these validated impact models in impact-aware robot manipulation schemes.

\section{ACKNOWLEDGMENTS}

The authors want to thank the Institut des Systèmes Intelligents et de Robotique (ISIR) at Sorbonne Universite and CNRS (Paris, France) for providing access to the KUKA LWR $\mathrm{IV}+$ robot used in this study, Lucas Joseph for collecting the collision data, and Claude Lacoursière, Maarten Jongeneel, and Wouter Weekers for valuable feedback on a preliminary draft of this document.

\section{REFERENCES}

[1] S.S.M. Salehian and A. Billard. A dynamical-system-based approach for controlling robotic manipulators during noncontact/contact transitions. IEEE Robotics and Automation Letters, 3(4):27382745, 2018

[2] A. Albu-Schffer, A. Bicchi, Actuators for Soft Robotics, in Springer Handbook of Robotics, 2nd edition S. Bruno, and O. Khatib eds., 499-530, 2016

[3] N.G. Tsagarakis, D.G. Caldwell, F. Negrello, W. Choi, L. Baccelliere, V.G. Loc, J. Noorden, L. Muratore, A. Margan, A. Cardellino, L. Natale, E. Mingo Hoffman, H. Dallali, N. Kashiri, J. Malzahn, J. Lee, P. Kryczka, D. Kanoulas, M. Garabini, M. Catalano, M. Ferrati, V. Varricchio, L. Pallottino, C. Pavan, A. Bicchi, A. Settimi, A. Rocchi, A. Ajoudani, WALK-MAN: A High-Performance Humanoid Platform for Realistic Environments, Journal of Field Robotics, 34(7):12251259, 2017

[4] J. Englsberger, A. Werner, C. Ott, B. Henze, M. A. Roa, G. Garofalo, R. Burger, A. Beyer, O. Eiberger, K. Schmid, A. Albu-Schffer, Overview of the torque-controlled humanoid robot TORO, 2014 IEEERAS International Conference on Humanoid Robots, 916-923, 2014

[5] M. Rijnen, E. de Mooij, S. Traversaro, F. Nori, N. van de Wouw, A. Saccon, H. Nijmeijer, Control of humanoid robot motions with impacts: Numerical experiments with reference spreading control. IEEE International Conference on Robotics and Automation (ICRA), 4102-4107, 2017

[6] Y. Wang, A. Kheddar, Impact-Friendly Robust Control Design with Task-Space Quadratic Optimization, Robotics: Science and Systems (RSS), 2019

[7] M. Halm, M. Posa, Modeling and Analysis of Non-unique Behaviors in Multiple Frictional Impacts. Robotics: Science and Systems (RSS), 2019

[8] Nima Fazeli, Samuel Zapolsky, Evan Drumwright, Alberto Rodriguez, Fundamental Limitations in Performance and Interpretability of Common Planar Rigid-Body Contact Models. International Symposium of Robotic Research (ISRR), 2017
[9] Y.-F. Zheng and H. Hemami, Mathematical modeling of a robot collision with its environment, Journal of Field Robotics, vol. 2, no. 3, pp. 289307, 1985

[10] J. K. Mills, "Manipulator transition to and from contact tasks: a discontinuous control approach," IEEE International Conference on Robotics and Automation (ICRA), pp. 440-446, 1990

[11] Y. Hurmuzlu, D.B. Marghitu, Rigid Body Collisions of Planar Kinematic Chains with Multiple Contact Points, International Journal of Robotic Research, 13(1):82-92, 1994

[12] D. Walker, Impact configurations and measures for kinematically redundant and multiple armed robot systems, IEEE Transactions on Robotics and Automation, vol. 10, no. 5, pp. 670-683, Oct. 1994

[13] D. N. Nenchev and K. Yoshida, Impact analysis and post-impact motion control issues of a free-floating Space robot subject to a force impulse. IEEE Transactions on Robotics and Automation, vol. 15, no. 3, pp. 548-557, June 1999

[14] H.W. Park, K. Sreenath, J. W. Hurst, J.W. Grizzle, Identification of a Bipedal Robot With a Compliant Drivetrain: Parameter Estimation for Control Design. Control Systems Magazine, 31(2):63-88, 2011

[15] Y.-B. Jia, M. Gardner, X. Mu, Batting an in-flight object to the target. The International Journal of Robotics Research, 38(4), 451485, 2019

[16] M. Rijnen, A. Saccon, H. Nijmeijer, Reference Spreading: Tracking Performance for Impact Trajectories of a 1DoF Setup, IEEE Transactions on Control Systems Technology, vol. 28, no. 3, pp. 1124-1131, May 2020

[17] J. Hollerbach, W. Khalil, M. Gautier, Model Identification, in Springer Handbook of Robotics, 2nd edition, S. Bruno, and O. Khatib eds., $113-138,2016$

[18] A. De Luca, W.J. Book, Robots with Flexible Elements in Springer Handbook of Robotics, 2nd edition S. Bruno, and O. Khatib eds., 243-282, 2016

[19] P.I. Corke, Robotics, Vision \& Control. Springer 2017

[20] B. Brogliato, Nonsmooth mechanics: models, dynamics and control. Third edition, Springer, 2016

[21] S. Haddadin, A. Albu-Schffer, G. Hirzinger, Safety Evaluation of Physical Human-Robot Interaction via Crash-Testing. Robotics: Science and Systems (RSS), 217-224, 2007

[22] Requirements for safe robots: Measurements, analysis and new insights S Haddadin, A Albu-Schffer, G Hirzinger. The International Journal of Robotics Research, 28(11-12):1507-1527, 2009

[23] S. Haddadin, A. Albu-Schaffer, A. De Luca, G. Hirzinger, Collision detection and reaction: A contribution to safe physical human-robot interaction. IEEE/RSJ International Conference on Intelligent Robots and Systems (IROS), 3356-3363, 2008

[24] N.S. Nguyen, B. Brogliato, Comparisons of multiple-impact laws for multibody systems: Moreaus law, binary impacts, and the LZB approach. Advanced Topics in Nonsmooth Dynamics, 1-45, 2018

[25] M. Moll, M.A. Erdmann, Manipulation of pose distributions. International Journal of Robotics Research, 21(3):277292, 2002

[26] J.W. Grizzle, C. Chevallereau, R.W. Sinnet, A.D. Ames, Models, feedback control, and open problems of 3D bipedal robotic walking. Automatica 50 (8), 1955-1988, 2014

[27] J. Reher, E. A. Cousineau, A. Hereid, C. M. Hubicki, A. D. Ames, Realizing dynamic and efficient bipedal locomotion on the humanoid robot DURUS, IEEE Int. Conf. on Robotics and Automation, pp. 17941801, 2016

[28] C. Glocker, An Introduction to Impacts. In Nonsmooth Mechanics of Solids, eds J.Haslinger and G.E. Stavroulakis, pp. 45-101, Springer, 2006

[29] L. Joseph and V. Padois and G. Morel. Towards X-ray medical imaging with robots in the open: safety without compromising performances. IEEE International Conference on Robotics and Automation, 66046610,2018

[30] L. Joseph and V. Padois and G. Morel. Experimental validation of an energy constraint for a safer collaboration with robots. International Symposium on Experimental Robotics, 2018

[31] K. H. Hunt and F. R. E. Crossley, Coefficient of Restitution Interpreted as Damping in Vibroimpact. ASME Journal of Applied Mechanics, 42(2): 440445, 1975

[32] R.M. Alexander, Three Uses for Springs in Legged Locomotion, The International Journal of Robotics Research, 9(2):53-61, 1990

[33] M. Rijnen, A. Saccon, H. Nijmeijer, Motion Signals With Velocity Jumps: Velocity Estimation Employing Only Quantized Position Data. IEEE Robotics and Automation Letters, 3(2):1498-1505, 2018 